This is a provisional PDF only. Copyedited and fully formatted version will be made available soon.

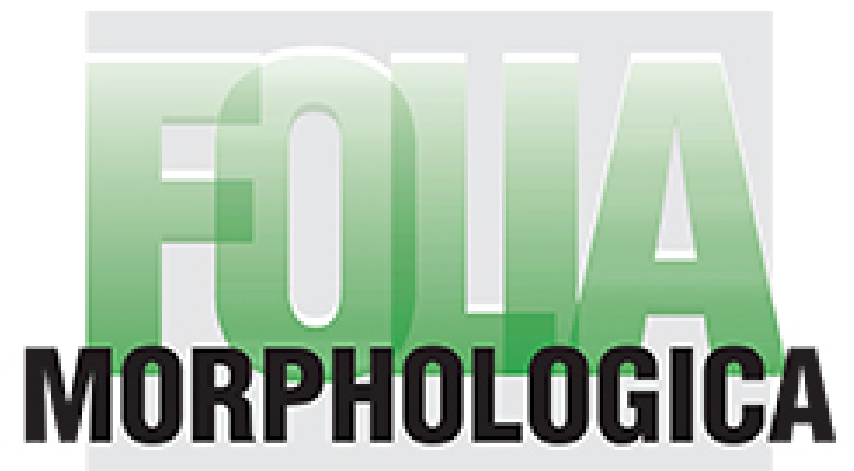

ISSN: 0015-5659

e-ISSN: $1644-3284$

\title{
Case report of the double headed extensor hallucis longus
}

Authors: K. Ruzik, K. Westrych, R. S. Tubbs, Ł. Olewnik, P. Łabętowicz, N. Zielinska

DOI: 10.5603/FM.a2022.0018

Article type: Case report

Submitted: $2021-10-28$

Accepted: 2021-11-30

Published online: 2022-02-17

This article has been peer reviewed and published immediately upon acceptance.

It is an open access article, which means that it can be downloaded, printed, and distributed freely, provided the work is properly cited.

Articles in "Folia Morphologica" are listed in PubMed. 


\title{
Case report of the double headed extensor hallucis longus
}

K. Ruzik et al., Case report of the double headed extensor hallucis longus

K. Ruzik ${ }^{1}$, K. Westrych ${ }^{1}$, R.S. Tubbs ${ }^{2-5}$, Ł. Olewnik ${ }^{1}$, P. Łabętowicz ${ }^{6}$, N. Zielinska ${ }^{1}$

${ }^{1}$ Department of Anatomical Dissection and Donation, Medical University of Lodz, Poland

${ }^{2}$ Department of Neurosurgery, Tulane University School of Medicine, New Orleans, LA,

United States

${ }^{3}$ Department of Neurosurgery and Ochsner Neuroscience Institute, Ochsner Health System, New Orleans, LA, United States

${ }^{4}$ Department of Anatomical Sciences, St. George’s University, Grenada

${ }^{5}$ Department of Surgery,Tulane University School of Medicine, New Orleans, LA, United States

${ }^{6}$ Department of Normal and Clinical Anatomy, Medical University of Lodz, Poland

Address for correspondence: Kacper Ruzik, MD, Department of Anatomical Dissection and Donation, Medical University of Lodz, ul. Żeligowskiego 7/9, 90-752 Łódź, Poland, e-mail: kacper.ruzik@umed.lodz.pl

\begin{abstract}
:
Background: We present a case report of double-headed extensor hallucis longus (EHL) with potential clinical significance.

Materials and methods: Cadaveric dissection of the right lower limb of a 70-year-old female at death was performed for research and teaching purposes at the Department of Anatomical Dissection and Donation, Medical University of Lodz. The limb was dissected using standard
\end{abstract}


techniques according to a strictly specified protocol. Each head and tendon of the muscle was photographed and subjected to further measurements.

Results: During dissection, an unusual type of EHL muscle was observed. It consisted of two muscle bellies, a main tendon and an accessory tendon. Both muscle bellies were located on anterior surface of the fibula and the interosseous membrane. The main tendon insertion was located on the dorsal aspect of the base of the distal phalanx of the big toe, while the accessory tendon insertion was located medially.

Conclusions: The EHL muscle is highly morphologically variable at both the point of origin and the insertion. Knowledge of its variationsis connected to several pathologies such as foot drop, tendonitis, tendon rupture, and anterior compartment syndrome.

Key words: extensor hallucis longus, additional muscle belly, anatomical variations, hallux valgus, embryology

\section{INTRODUCTION}

The extensor hallucis longus (EHL), in addition to the tibialis anterior (TA) and extensor digitorum longus (EDL), is part of the anterior compartment of the lower limb.[24, 35] Its origin is located on the anterior surface of the fibula and on the interosseous membrane lateral to the origin of the TA and medial to the origin of the extensor digitorum longus. The EHL is situated in the middle of these muscles and is largely covered by them. [17]

The thin muscle belly becomes the tendon situated behind the superior and inferior extensor retinaculum and is then inserted into the dorsal aspect of the base of the distal phalanx of the big toe. [38] The EHL is innervated by branches originating from the deep fibular nerve and is supplied by branches of the anterior tibial artery. [2, 34]

The role of the EHL is to extend the great toe. It is also responsible for dorsiflexion of the foot, adjunct foot eversion and inversion.There are EHL classifications for fetuses and adults, and more recently an exact classification was proposed by Zielinska et al.[1, 7, 17, 22, 26, 27, 38], distinguishing three types of EHL with subtypes. Anatomical variations of the 
EHL can predispose to hallux valgus deformity, tendonitis and acute compartment syndrome. $[11,16,33]$.

The present report describes a case of a two-headed EHL. Knowledge of the morphological variability of this muscle is significant for orthopedic surgeons, physiotherapists and anatomists.[17, 27]

\section{CASE REPORT}

During a routine dissection performed for research and teaching purposes at the Department of Anatomical Dissection and Donation, Medical University of Lodz, a supernumerary head of the EHL was found. (Fig. 1)The dissection was performed using traditional anatomical methods according to a previously described protocol.[17, 24, 27]. The subject of the dissection was a 70-year-old at death female cadaver.

The right lower limb was positioned in the supine position on the dissection table. First, all the skin,subcutaneous tissue and superficial fascia were removed up to the crural region and from the area of the foot. Secondly, the crural fascia was then carefully removed. Then the muscle bellies and tendons were cleaned from the medial to lateral side. After that, the muscles of the anterior crural compartment were separated from each other. Finally, the tendons were dissected to identify potential additional tendon bands.

Each head of the muscle and each tendon was photographed and measured using an electronic caliper with an accuracy of up to $0.1 \mathrm{~mm}$ (Mitutoyo Corporation, Kawasaki-shi, Kanagawa, Japan), each measurement being performed twice by two researchers.

The dominant muscle belly originated from the anterior surface of the fibula and from the interosseous membrane $208.45 \mathrm{~mm}$ above a line drawn between the malleoli. The width and thickness of the muscle were $19.67 \mathrm{~mm} \times 3.55 \mathrm{~mm}$ at the origin. The length of the muscle belly was $167.88 \mathrm{~mm}$.The width and thickness of the muscle were $4.27 \mathrm{~mm} \times 1.91 \mathrm{~mm}$ upon passing the muscle belly.

The lesser muscle belly appeared from beneath the anterior surface of the fibula and from the interosseous membrane $83.44 \mathrm{~mm}$ above a line drawn between the malleoli. The width and thickness of the muscle were $12.55 \mathrm{~mm} \times 2.30 \mathrm{~mm}$ at the origin. The length of the 
muscle belly was $87.02 \mathrm{~mm}$. The width and thickness of the muscle were $3.32 \mathrm{~mm} \times 1.03 \mathrm{~mm}$ upon passing the muscle belly.

The type of insertion corresponds to Subtype 2B proposed by Zielinska et al. [38] The main tendon length was $184.60 \mathrm{~mm}$. The width and thickness of the tendon were $5.63 \mathrm{~mm} \times$ $0.95 \mathrm{~mm}$ at the insertion, which was located on the dorsal aspect of the base of the distal phalanx of the big toe. The additional tendon length was $119.59 \mathrm{~mm}$. The width and thickness of the tendon were $2.33 \mathrm{~mm} \times 0.42 \mathrm{~mm}$ at the insertion, which was located on the dorsal aspect of the proximal phalanx of the hallux, medial to the insertion of the EHB main tendon.

No extension point (i.e., the point where the tendon begins to expand into its insertional footprint) was observed at any of the described tendons.

\section{DISCUSSION}

Accessory muscle bellies inthe upper and lower limbs have been widely reported.Such anatomical variations are common among large muscles such as the quadriceps femoris, coracobrachialis, biceps brachii, subscapularis and also vestigial muscles such as the plantaris. [15, 29, 32, 36, 37] An accessory muscle head can be related to potential compression of nerves or vessels but can be useful during reconstruction surgery.[25, 36]Whether additional muscle bellies are rare anatomical variations or are more often observed thanks to more carefully performed studies and better embalming of human cadavers is an open question. For example, Olewnik et al.[28] found an additional head of the quadriceps femoris muscle in 68 out of 106 lower limbs and opened the discussion: is it quadriceps or multiceps femoris?

\section{Evolutionary aspect}

The EHL, despite the quadratus plantae and plantaris muscles, appeared first in Mammalia.[8, 18]In all hominoids, it originates from the medial aspect of the fibula shaft, and in Hylobates and Pan there is an additional origin from the lateral condyle of the tibia. [6] In all taxa, insertion is onto the dorsum of the distal phalanx of the hallux. However, Ferrero et al. reported an additional insertion onto the dorsum of the proximal phalanx in a gorilla limb. [12] Origins from the interosseous membrane of the leg have also been demonstrated in the gorilla. Interestingly, Ferrero et al. only reported this type of proximal attachment in a single orangutan limb.[12]It was not described among apes.

\section{Embryological aspect}


To understand the complexity of the variations of the EHL insertion, it is necessary to considerits embryological basis. In a five-week embryo,the limbs start to form when the mesoderm migrates into the limb bud and anterior and posterior condensations start to generate the musculoskeletal system of the lower limb.[3, 4] The posterior condensation develops into the extensor musculature of the lower limb. The EHL splits from the deep portion of thisextension mass, although its tendon remains fused with the extensor digitorum longus, with which it is often fused in later life. [13, 20, 21]

Retinoic acid, sonic hedgehog (SHH), HOX genes (Hoxd-9, Hoxd-10, Hoxd-11, Hoxd-12, Hoxd-13), the apical ectodermal ridge (AER), and the zone of polarizing activity (ZPA) are described as factors responsible for forming the lower limb musculature. Aberrant gene expressions can cause malformations ofthe limbs. [5]

\section{Morphological variability}

Anatomy textbooks describe the insertion of the EHL into the dorsum of the base of the distal phalanx of the big toe. However, severalstudieshave described variations of this insertion.[1, 7, 17, 22, 26, 27]

Al-Sagaff described three types of possible distal attachment. [1]Type I (most common) was characterized as a single tendinous insertion on the dorsal aspect of the base of the distal phalanx of the big toe. In subsequent years, new classifications based on adult cadavers, fetuses and ultrasonography were proposed. [1, 7, 9, 17, 22, 26, 27] The most recent classification proposed by Zielinska et al. [38] summarizes the previously mentioned studies. They proposed three types of EHL insertion.

The proximal attachment of the EHL is less varied than the insertion. Egea et al. described a case report of a double origin of the EHL.[10] In contrast to our study they reported a medial and lateral origin of this muscle; we present upper and lower muscle bellies with separate innervations.

\section{Clinical significance}

The EHL tendon has been used in a variety of tendon transfer and tenodesis surgeries to correct iatrogenic hallux varus deformity, equinovarus foot deformity, clawed hallux associated with a cavus foot, and dynamic hyperextension of the hallux though it is usually used to prevent pedal imbalance after transmetatarsal amputation. [16, 31]

Importantly, rupture or significant injury to the EHL tendon can result in a dropped hallux, loss of dorsiflexion, resulting in an unpaired gait.[11] This can be treated surgically by 
transferring the first slip of the extensor digitorum longus and extensor hallucis brevis to the injured EHL. Rupture of this tendon is a result of laceration. [11]

The EHL is also implicated in tendonitis, when the tendon becomes overstressed causing inflammation and pain. [23]Excessive calf muscle tightness, overextension during exercise, and falling of the foot arch are listed as the most common factors. [23]The condition can be treated with stretching, NSAIDs, and walking boots in more severe cases. [19]

The anterior compartment of the leg is also the most common site for acute compartment syndrome, which can result from trauma, blunt force injury, or reperfusion after acute interruption of flow to muscles in the crural compartment. Swelling after these insults can lead to muscle ischemia because the muscles in these compartments are highly bound by the tough crural fascia, making it difficult for them to expand in response to injury. When the muscles expand they can compress the artery, resulting in ischemic injury. Nerves can also be compressed causing loss of neuromuscular function. Importantly, definitive management of acute compartment syndrome is a subcutaneous fasciotomy. [33] There is also chronic compartment syndrome resulting from excessive exercise, causing pain and swelling. The pressure is increased by increases in blood flow and muscle mass from excessive exercise. As a result, arteries can be compressed.[33] It is feasible that an additional muscle belly will aggravate the symptoms of both acute and chronic compartment syndrome and be another anatomical structure that can compress nerves or vessels.

The hallux valgus deformity is a common forefoot pathology. It is characterized by lateral deviation of the great toe (hallux) and medial deviation of the first metatarsal and has several possible causes, the most common being wrongly matched footwear. [38]Other factors include muscle imbalance, genetic predisposition, ligamentous laxity and long-standing work in an upright posture. [30]However, the involvement of the EHL in hallux valgus pathogenesis is unclear. Natsis et al. found that hallux valgus coincided with an accessory tendon of the EHL in $65.4 \%$ of the feet examined. If additional tendons predispose to hallux valgus deformity, it seems likely that a second muscle belly also affects the developmentof this pathology. [22]However, biomechanical research is needed to confirm this prediction.

\section{CONCLUSIONS}

The extensor hallucis longus muscle is highly morphologically variable at both the points of origin and insertion. Knowledge of its variations is related to several pathologies such as foot drop, tendonitis, tendon rupture, and anterior compartment syndrome. 


\section{Acknowledgments}

The authors sincerely thank those who donated their bodies to science so that anatomical research could be performed. Results from such research can potentially increase mankind's overall knowledge that can then improve patient care. Therefore, these donors and their families deserve our highest gratitude [14].

The authors wish to express their gratitude to all those who donated their bodies to medical science.

\section{Declarations and Ethical approval}

The cadavers were the property of the Department of Anatomical Dissection and Donation, Medical University of Lodz,

This article describes no studies involving human participants or animals performed by any of the authors.

Conflict of interest: None declared

\section{REFERENCES}

1. Al-Saggaf S (2003) Variations in the insertion of the extensor hallucis longus muscle. Folia Morphol (Warsz) 62:147-155

2. Aragonés P, Rodríguez-Niedenführ M, Quinones S, de Blas CS, Konschake M, Sanudo JR, Vázquez MT (2021) Popliteal artery: Anatomical study and review of the literature. Ann Anat = Anat Anzeiger Off organ Anat Gesellschaft 234:151654. doi: 10.1016/j.aanat.2020.151654

3. Bardeen CR (1905) Studies of the development of the human skeleton. (A). The development of the lumbab, sacbal and coccygeal vertebwe. (B). The cubves and the pbopobtionate regional lengths of the spinal column during the first thbee months of embbyonic developnent. (C). T. Am J Anat 4:265-302. doi: https://doi.org/10.1002/aja.1000040302

4. Bardeen CR (1906) Development and variation of the nerves and the musculature of the inferior extremity and of the neighboring regions of the trunk in man. Am J Anat 6:259-390. doi: https://doi.org/10.1002/aja.1000060108

5. Barham G, Clarke NMP (2008) Genetic regulation of embryological limb development with relation to congenital limb deformity in humans. J Child Orthop 2:1-9. doi: 10.1007/s11832008-0076-2 
6. Beddard FE (1893) VII. Contributions to the Anatomy of the Anthropoid Apes. Trans Zool Soc London. doi: 10.1111/j.1096-3642.1893.tb00049.x

7. Bibbo C, Arangio G, Patel D V (2004) The accessory extensor tendon of the first metatarsophalangeal joint. Foot ankle Int 25:387-390. doi: 10.1177/107110070402500604

8. Boyer EL (1935) The musculature of the inferior extremity of the orang-utan Simia satyrus. Am J Anat 56:193-256. doi: https://doi.org/10.1002/aja.1000560203

9. Denk C, Öznur A, Sürücü H (2002) Double tendons at the distal attachment of the extensor hallucis longus muscle. Surg Radiol Anat 24:50-52. doi: 10.1007/s00276-002-0016-7

10. Egea JM, Cabeza L, Ortiz R, Rama AR, Melguizo C, Prados J (2019) Double origin of the extensor hallucis longus muscle: a case report. Surg Radiol Anat 41:1421-1423. doi: 10.1007/s00276-019-02309-5

11. Fadel GE, Alipour F (2008) Rupture of the extensor hallucis longus tendon caused by talar neck osteophyte. Foot ankle Surg Off J Eur Soc Foot Ankle Surg 14:100-102. doi: 10.1016/j.fas.2007.11.004

12. Ferrero EM, Pastor F, FERNANDEZ F, Barbosa M, Diogo R, WOOD B (2012) Comparative anatomy of the lower limb muscles of hominoids: attachments, relative weights, innervation and functional morphology.

13. Gros J, Tabin CJ (2014) Vertebrate limb bud formation is initiated by localized epithelial-tomesenchymal transition. Science 343:1253-1256. doi: 10.1126/science.1248228

14. Iwanaga J, Singh V, Ohtsuka A, Hwang Y, Kim H-J, Moryś J, Ravi KS, Ribatti D, Trainor PA, Sañudo JR, Apaydin N, Şengül G, Albertine KH, Walocha JA, Loukas M, Duparc F, Paulsen F, Del Sol M, Adds P, Hegazy A, Tubbs RS (2021) Acknowledging the use of human cadaveric tissues in research papers: Recommendations from anatomical journal editors. Clin Anat 34:24. doi: 10.1002/ca.23671

15. Je S-S, Park B, Kim J, Yoon S-P (2016) Five-headed biceps brachii muscle with a rare origin from the tendon of pectoralis major muscle. Anat Sci Int 91:110-113. doi: 10.1007/s12565015-0288-8

16. Johnson KA, Spiegl P V (1984) Extensor hallucis longus transfer for hallux varus deformity. J Bone Joint Surg Am 66:681-686

17. Karauda P, Shane Tubbs R, Polguj M, Olewnik Ł (2021) Morphological variability of the extensor hallucis longus in human fetuses. Ann Anat 234:151627. doi:

10.1016/j.aanat.2020.151627

18. Lewis OJ (1966) The phylogeny of the cruropedal extensor musculature, with special reference to the primates. J Anat

19. Lezak B, Summers S (2021) Anatomy, Bony Pelvis and Lower Limb, Extensor Hallucis Longus Muscle. Treasure Island (FL)

20. Lezak B, Wehrle CJ, Summers S (2020) Anatomy, Bony Pelvis and Lower Limb, Posterior Tibial Artery. StatPearls Publishing, Treasure Island (FL) 
21. Mróz I, Kielczewski S, Pawlicki D, Kurzydło W, Bachul P, Konarska M, Bereza T, Walocha K, Kaythampillai LN, Depukat P, Pasternak A, Bonczar T, Chmielewski P, Mizia E, Skrzat J, Mazur M, Warchoł Ł, Tomaszewski K (2016) Blood vessels of the shin - anterior tibial artery anatomy and embryology - own studies and review of the literature. Folia Med Cracov 56:3347

22. Natsis K, Konstantinidis GA, Symeonidis PD, Totlis T, Anastasopoulos N, Stavrou P (2017) The accessory tendon of extensor hallucis longus muscle and its correlation to hallux valgus deformity: a cadaveric study. Surg Radiol Anat 39:1343-1347. doi: 10.1007/s00276-017-18814

23. Ng JM, Rosenberg ZS, Bencardino JT, Restrepo-Velez Z, Ciavarra GA, Adler RS (2013) US and MR Imaging of the Extensor Compartment of the Ankle. RadioGraphics 33:2047-2064. doi: 10.1148/rg.337125182

24. Olewnik $€$ (2019) Fibularis Tertius: Anatomical Study and Review of the Literature. Clin Anat 32:1082-1093. doi: 10.1002/ca.23449

25. Olewnik Ł, Paulsen F, Tubbs RS, Zielińska N, Szewczyk B, Karauda P, Polguj M (2021) Potential compression of the musculocutaneous, median and ulnar nerves by a very rare variant of the coracobrachialis longus muscle. Folia Morphol (Warsz) 80:707-713. doi: 10.5603/fm.a2020.0085

26. Olewnik Ł, Podgórski M, Polguj M, Ruzik K, Grzelak P (2020) Is ultrasound effective in determining variation of the insertion of the extensor hallucis longus tendon? Clin Anat 33:1235-1239. doi: 10.1002/ca.23572

27. Olewnik Ł, Podgórski M, Polguj M, Ruzik K, Topol M (2019) A cadaveric study of the morphology of the extensor hallucis longus - a proposal for a new classification. BMC Musculoskelet Disord 20:310. doi: 10.1186/s12891-019-2688-8

28. Olewnik Ł, Tubbs RS, Ruzik K, Podgórski M, Aragonés P, Waśniewska A, Karauda P, Szewczyk B, Sanudo JR, Polguj M (2021) Quadriceps or multiceps femoris?_Cadaveric study. Clin Anat 34:71-81. doi: 10.1002/ca.23646

29. Olewnik Ł, Zielinska N, Karauda P, Tubbs RS, Polguj M (2020) A three-headed plantaris muscle: evidence that the plantaris is not a vestigial muscle? Surg Radiol Anat 42:1189-1193. doi: $10.1007 / \mathrm{s} 00276-020-02478-8$

30. Perera AM, Orth F, Mason L, Eng M, Stephens MM (2011) The Pathogenesis of Hallux Valgus. 1650-1661

31. Roukis TS (2009) Flexor hallucis longus and extensor digitorum longus tendon transfers for balancing the foot following transmetatarsal amputation. J foot ankle Surg Off Publ Am Coll Foot Ankle Surg 48:398-401. doi: 10.1053/j.jfas.2008.12.013

32. Ruzik K, Waśniewska A, Olewnik Ł, Tubbs RS, Karauda P, Polguj M (2020) Unusual case report of seven-headed quadriceps femoris muscle. Surg Radiol Anat 42:1225-1229. doi: 10.1007/s00276-020-02472-0

33. Via AG, Oliva F, Spoliti M, Maffulli N (2015) Acute compartment syndrome. Muscles Ligaments Tendons J 5:18-22 
34. Vittoria N, Giuseppe M, Ivano D, Giovanni B (2009) The innervation of extensor hallucis longus muscle: An anatomical study for selective neurotomy. Acta Neurochir (Wien) 151:1275-1279. doi: 10.1007/s00701-009-0504-1

35. Zdilla MJ, Paulet JE, Lear JJ, Addie KM, Lambert HW (2018) A Review of Extensor Hallucis Longus Variants Featuring a Novel Extensor Primi Internodii Hallucis Muscle Merging With Extensor Hallucis Brevis. J Foot Ankle Surg 57:1218-1220. doi: 10.1053/j.jfas.2018.03.031

36. Zielinska N, Olewnik Ł (2021) Six-headed coracobrachialis muscle. Folia Morphol (Warsz). doi: 10.5603/fm.a2021.0082

37. Zielinska N, Olewnik Ł, Karauda P, Tubbs RS, Polguj M (2021) A very rare case of an accessory subscapularis muscle and its potential clinical significance. Surg Radiol Anat 43:1925. doi: 10.1007/s00276-020-02531-6

38. Zielinska N, Tubbs RS, Ruzik K, Olewnik $€$ (2021) Classifications of the extensor hallucis longus tendon variations: Updated and comprehensive narrative review. Ann Anat 238:151762. doi: 10.1016/j.aanat.2021.151762

Figure 1. EHL- Extensor hallucis longus, White star- main mussle belly, Green staradditional muscle belly, EDL- Extensor digitorum longus, TAT- Tibialis anterior tendon 


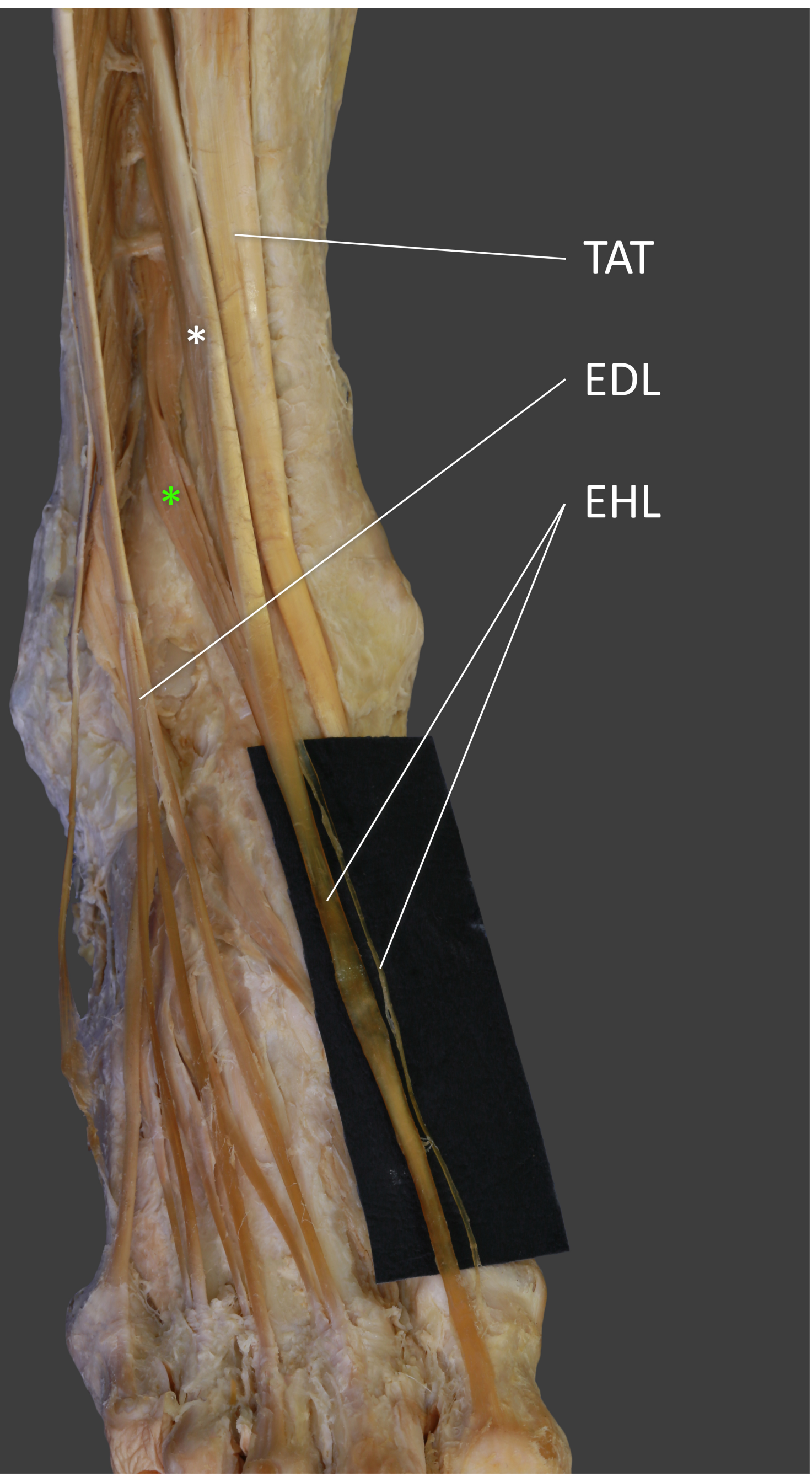

